\title{
Cultural Development of Dongying City Driven by Lv Opera from the Perspective of Urban Sustainability
}

\author{
Xu Wang ${ }^{1, *} ;$ Wenxin Yuan ${ }^{2}$ \\ ${ }^{1}$ School of Literature, Law and Business, Shengli College China University of Petroleum, Dongying, Shandong 257061, \\ China \\ ${ }^{2}$ School of Literature, Law and Business, Shengli College China University of Petroleum, Dongying, Shandong 257061, \\ China \\ *Corresponding author. Email: 1453454677@qq.com
}

\begin{abstract}
In this age of fast urbanization and social development, urban culture has seen tectonic changes. Sustainable development of culture becomes a salient issue that stems further urban development. With Lv opera of Dongying City as the study object, this paper probes into the factors that influence the healthy development of urban culture. The first section analyzes the economic status of cities, and starting from the Lv opera culture and urban culture, interprets the necessity of sustainable development of urban culture; the second section analyzes the causes for low matching degree between Lv opera and urban culture from the angle of cultural development; the third section proposes suggestions for sustainable development of urban culture in Dongying City.

Keywords: urban sustainability, Lv opera culture, urban culture
\end{abstract}

\section{Value of Lv opera in Dongying City and status quo of urban sustainable development}

\subsection{Cultural value of Lv opera}

Lv opera is the cultural heritage of people in Shandong, especially in Dongying City; it is a carrier of tradition and provides a graceful way of entertainment. With its unique value and sentimental connotation, Lv opera reflects the traditional life style and spirit of people in Shandong, especially in Dongying City, and manifests the urban culture.

\subsection{Analysis of urban sustainability development}

Urban sustainability development means realizing rapid urbanization and modernization of a city by way of longterm and continuous growth and structuring of the city under a given tempo-spatial dimension so as to meet the increasing needs of development. This paper focuses on the sustainable development of culture in Dongying City so that the urban culture can meet the increasing cultural needs and future development. [1]

\subsection{Lv opera culture and sustainable development of Dongying City}

As the global culture diversifies and urban culture progresses, many traditional culture forms, however, remains unchanged and fails to keep up with the pace of urban development. [2] Lv opera, an important part of traditional culture, is a unique form of local opera in Shandong province. After passing its prime, heritage and development of this opera is now under impact from modern culture. "Old operas play over and over again", the manifestation lacks diversity, the target audience is limited and the Lv opera troupe is under pressure of getting dismantled. Therefore, the Lv opera needs to recreate its image to promote sustainable development of urban culture of Dongying City .

\section{Causes for mismatch between Lv opera culture and urban development in Dongying City}

\subsection{Root causes for mismatch between $\mathrm{Lv}$ opera culture and urban development in Dongying City}

Lv opera has been its prime in the last century. After the Reform and Opening Up, under the impact of global culture, inheritance of opera culture has taken efforts from generations. Lv opera culture must keep up with the pace 
of time to avoid dismatch with the urban development. Therefore, how to maintain and develop the features of traditional culture and meanwhile adapt it to the new needs of urban cultural development is a major challenge in the initiative to promote urban cultural development with $\mathrm{Lv}$ opera culture, [3] and it is also a major challenge that Dongying City must face in its cultural development.

\subsection{Causes for mismatch between Lv opera culture and urban development of Dongying City}

\subsubsection{Modern lifestyle is dissolving traditional opera culture}

Dissolving of traditional opera culture, especially Lv opera in Dongying, by modern lifestyles is reflected in the following aspects: the first impact comes from new media; the monotonous stage performance-based form of $\mathrm{Lv}$ opera can no longer satisfy the artistic pursuit of the modern audience. The second factor is the changes in social culture. As the form of culture diversifies, the audience begin to turn their attention from Lv opera and traditional culture to other forms of modern culture; thirdly, with the advent of the age of fast consumption, the cultural consumption experience of the audience differs from the past, and the prevailing fickle mindset among the audience can no long appreciate the traditional culture that requires time to taste and absorb. Therefore, traditional Lv culture is dissolving by the fickle modern lifestyle.

\subsubsection{Mistmatch between the subject of culture inheritance and that of urban culture development}

Lack of a training system for Lv opera talents leads to the mismatch between the subject of culture inheritance and that of urban culture development - this accounts for an important reason why Lv opera culture fails to keep up with the pace of urban cultural development. After the elder opera masters retire, the young generation of $\mathrm{Lv}$ opera performers have not been trained [4], and as a result, no one shoulders the responsibility of inheriting $\mathrm{Lv}$ opera culture. [5]

Meanwhile, as education develops, most pays more attention to new culture than to traditional culture inheritance. The younger generations prefer culture that is more vibrant and promising. They are the new force for urban culture sustainable development and also the major force of different cultures. Therefore, there is a mismatch between urban culture developers and Lv opera culture inheritors, leading to a vicious cycle for inheritance of $\mathrm{Lv}$ opera culture. [5]

\subsubsection{Losing of the soil for urban culture}

The new-culture based soil of urban culture is no longer just for inheritance and development of traditional culture, but has shifted its focus to discovery of new culture. [6] In the fickle cultural environment in cities [7], new culture is trapped in a vicious cycle of fast emergence, rapid development and quick disappearance. Take cultural creation as an example. Any art has its own process of creation, and the creation of an episode of Lv opera [8] would consume time and energy of a few generations; the plot alone would take several years to develop, and the whole opera would take years of practice and performance before it became a classic. However, as the urban culture is rushing to keep up with the pace of globalization, the soil for traditional culture is encroached. [9]

\section{SUSTAINABLECONSTRUCTIONANDDEVELOPMENT OFURBANCULTURE}

Urban culture and traditional culture are closely connected and mutually dependent. As a unique form of culture of Dongying City, Lv opera culture is an indispensable part of sustainable development of the city. With innovation of urban culture, Lv opera culture has some artistic charm that no other cultures can match, but new forms are needed to display this unique charm. Therefore, for sustainable development of urban culture of Dongying City [10] , the following three suggestions are proposed:

\subsection{Co-development of culture and the city}

With the development of social economy, culture plays an increasingly important role, and the improvement of life quality leads to a higher demand for art appreciation [11] . Lv opera should feel the pulse of development and traditional culture should develop together with urban development so that culture can play a promoting role in the economy.

First, culture should be integrated into urban life. Billboards, slogans and other forms of publicity should be used to promote culture and allow culture to penetrate every corner of our life [12] . Besides, urban planning should take culture into consideration and in the process of urban development, the government should pay more attention to cultural development. The government should provide strong support for cultural development and put equal emphasis on culture and economy.

\subsection{Merge of tradition and technology}

Modern technologies have brought impacts to inheritance and development of traditional culture, but undeniably, traditional culture has to rely on modern technologies to achieve further development [13] . Take the culture resource protection, inheritance and development in 
Dongying City as an example. Modern ways of display and innovation introduces new charm to Lv opera and blazes a new trail for cultural development. In the meantime, scientific innovation is changing the nature and appearance of traditional culture. Therefore, to merge traditional Lv opera culture into the modern urban culture and realize sustainable development of urban culture, we must rely on technologies [14] .

First of all, we should update the promotion ways. Besides the old forms of stage performance, Lv opera culture should create new forms of display. For instance, we can make sue of new media like the Internet, media, TV, Weibo, WeChat, short-video platforms as the way to diversify promotion of Lv opera [15] . In the meantime, we should merge Lv opera with technology, expand the audience of Lv opera, making it an art not only for the old, but also for the young generations. We should make technology not only the label of the young, but also the label of the $\mathrm{Lv}$ opera, realizing integration of $\mathrm{Lv}$ opera with technology [16]

\subsection{Support from society and schools in inheritance}

The major force of urban culture development is the young, and the backup force is the young students in schools [17] . Therefore, to allow the Lv opera culture promote development of urban culture, we must seek support from the society and schools.

First of all, we should unlock the potential of the market and expand the audience. Lv opera theatres are the platforms for development, so they should play an increasing role. Theatres can lower the price of tickets to attract more audience and increase the passion of others for the Lv opera [18] . Besides, to solve the problem of lack of cultural inheritors, the government can organize Lv opera contests for the young people and provide incentives to draw participants.

Moreover, schools are the cradles of talents, so they can train Lv opera talents and introduce them to the society to realize sustainable development. Regional primary and secondary schools should support publicity campaigns of Lv opera culture and organize corresponding class activities. Support from the society and schools can provide a strong foundation for development of Lv opera, and in this way [19], Lv opera culture can be made fully use of and be integrated into the social culture. As a result, the urban culture and the Lv opera culture can both be raised to a new level of development.

\section{CONCLUSIONS}

The Lv opera embodies the long history of traditional culture and the spirit of the old generations. Sustainable development of Lv opera culture in Dongying City should rely on social support and inheritors of the culture. A city's sustainable development is manifested not only on the economy, but on the culture. The unique Lv opera culture of Dongying City fosters the unique urban culture [20] . Currently, the Lv opera culture is in conflict with urban culture, and this conflict can be resolved by coordination among economy, technology and people. Therefore, we should make full use of technologies to integrate Lv opera into urban development, rely on social support to introduce Lv opera to more audience, and rely on schools to train talents to inherit and develop this traditional form of art. With these measures, the Lv opera can play a contributing role to prosperity of Dongying City.

\section{FUND PROJECTS :}

key topics of art science in Shandong province in 2019 : A Study on the Coincidence and Popularization of Lv Opera Culture in Shandong Youth Group from the Perspective of New Media ( Project number: ZH201906004)

\section{REFERENCES}

[1]Urban Culture and Urban Modernization .[J]. Xiao Bin . Yangtze River Forum . 2000(05)

[2]Innovation of Regional Economic Development Path from the Perspective of Regional Culture .[J]. Li Hao.Reform and strategy . 2012(05)

[3]Discussion on resilient city and sustainable development from urban planning and construction.[J]. Zhou Mengqi.Modern Horticulture. 2019(12)

[4]Research on Urban Planning and Construction Based on the Concept of Sustainable Development.[J]. Qian Shuguang.Jiangxi Building Materials. 2017(11)

[5]Chinese Culture and Chinese Drama.[M]. Zheng Xianchun .Changsha: Hunan People's Publishing House . 2007

[6]Urban Culture: The Internal Drive of Urban Development. [J]. Zhang Hongmei .Beauty and the Times. 2018(11)

[7]Urban Culture and Urban Characteristics . [J].Cong Hui .Literature and History . 2010(06)

[8]Discussion on Multivariate Value of Intangible Cultural Heritage -- Shandong Luju as an Example .[J].Liu Hongyan,Wang Yu.Shandong Social Sciences .2010(07)

[9]Constructing Theory and Strategy of Urban Landscape Transformation Based on Cultural 
Field .[J].Feng Yan, Liu Chuanlong .City Development Research .2018(03)

[10] The Application of the Three Kingdoms Culture in the Visual Image Building of Xiangyang City .[J].Cao Shujin .Housing and Real Estate .2017(29)

[11]Analysis on Representation Structure and Construction Path of Soft Power of Urban Culture .[J]. Yang Zheng. Future and Development . 2014(09)

[12]Exploring the Urban Connotation: The Process and Paradigm of Chinese Urban Culture Research .[J]. Li Yajuan. Chinese Culture Forum . 2017(01)

[13]An Empirical Study on Using Taiji Culture Resources to Promote the Brand Building of Urban Culture -- Taking Tai Chi Rong Cheng as an Example .[J]. Chen Qian, Gong Maofu, Ai Zexiu. Chinese Martial Arts (Research) . 2017(09)

[14]Inheritance of Non-heritage Culture and Brand Building of Urban Culture -- Taking Ningbo City as an Example .[J]. Yao Chenxi, Zheng Xuan, Jiang Zhenyi. Modern marketing (next issue) . 2017(10)

[15]On the Development of Urban Image from Luoyang City Culture Construction .[J]. Wang Yan. Journal of Jilin Institute of Education . 2011(07)

[16]Interpretation and performance of Hess Culture: Study on the Brand Building Path of Quanzhou City under the Background of "Belt and Road" . [J]. Peng Zhijian. Journal of Qingdao University of Science and Technology (Social Sciences Edition) . 2018(01)

[17]The path and mode of the integration of science and technology and culture in the protection and renewal of historic blocks . [J].Tao Liping, Yu Yufan, Zhang Guochao. Journal of Wuhan University of Light Industry . 2018(01)

[18]A Study on the Creation and Dissemination of Urban Cultural Brands -- Taking the Building of "Tang Xianzu" in Fuzhou City as an Example .[J].Hou Lijuan. Construction of Old Areas .2016(24)

[19]A Comparative Study on the Competitiveness of Urban Cultural Industry Gathering in China .[J].Xiong Jianlian, Xiao Chubo, Ren Yinghua. Statistics and decision-making .2017(01)

[20] A Study on the Strategies of Urban Cultural Construction from the Perspective of New Media -Taking Chuzhou City as an Example .[J].Zhu Jinxin, Jincan. Modern Decoration (Theory) .2017(01) 